livraisons

d'Histoire

de l'Architecture

\section{Livraisons de l'histoire de l'architecture}

27 | 2014

Varia II

\title{
Fiat justitia. Le palais de justice de Strasbourg (1892
}

- 1898)

Fiat justitia, The Court House of Strasbourg (1892-1898)

Fiat justicia. Der Straßburger Justizpalast (1892-1898)

Philippe Grandvoinnet et Raphaël Labrunye

\section{OpenEdition}

\section{Journals}

Édition électronique

URL : http://journals.openedition.org/lha/568

DOI : $10.4000 /$ lha. 568

ISSN : 1960-5994

Éditeur

Association Livraisons d'histoire de l'architecture - LHA

Édition imprimée

Date de publication : 10 juin 2014

Pagination : 51-61

ISSN : 1627-4970

Référence électronique

Philippe Grandvoinnet et Raphaël Labrunye, «Fiat justitia. Le palais de justice de Strasbourg (1892 -

1898) », Livraisons de l'histoire de l'architecture [En ligne], 27 | 2014, mis en ligne le 10 juin 2016,

consulté le 19 avril 2019. URL : http://journals.openedition.org/lha/568 ; DOI : 10.4000//ha.568

Ce document a été généré automatiquement le 19 avril 2019

Tous droits réservés à l'Association LHA 


\title{
Fiat justitia. Le palais de justice de Strasbourg (1892 - 1898)
}

\author{
Fiat justitia, The Court House of Strasbourg (1892-1898) \\ Fiat justicia. Der Straßburger Justizpalast (1892-1898)
}

\section{Philippe Grandvoinnet et Raphaël Labrunye}

1 Le palais de Justice de Strasbourg occupe une place singulière dans le plan de la Neustadt de Strasbourg. Ce quartier, édifié par les Allemands à partir de 1870 pour étendre la capitale alsacienne au nord, est en effet conçu selon une composition monumentale formée par l'axe reliant l'Université à la Kaiserplatz (actuelle place de la République). Programmé plus tardivement que les autres grands édifices publics du quartier, le palais de Justice se situe aux marges de la composition urbaine. Afin de relier visuellement l'église Saint-Pierre-le-Jeune (réalisée en 1895, ill. 1) au Palais de l'Empereur, une rupture géométrique va être introduite dans le tracé des voies.

III. 1 : Vue générale du quai Finkmatt, de gauche à droite : le palais de justice, l'église saint-Pierrele-Jeune catholique, la place et le canal

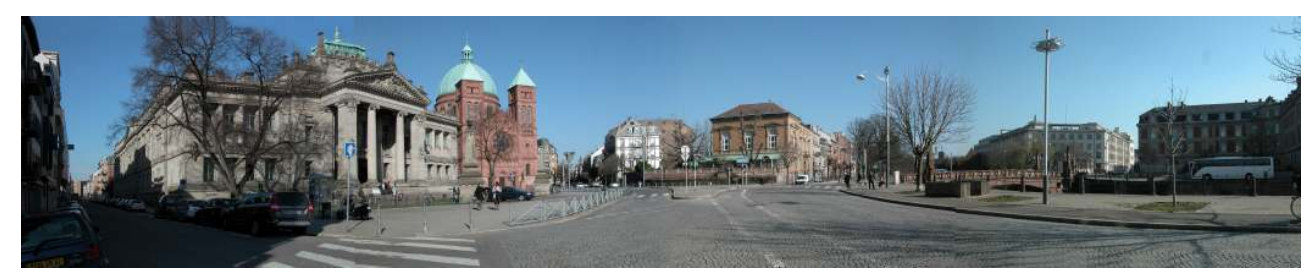

Photographie : Philippe Grandvoinnet, 2011

Cette évolution correspond aux modifications qui vont s'opérer progressivement dans la Neustadt dans un esprit plus pittoresque. Juxtaposé à l'église, le palais de Justice tente d'articuler cette grande composition allemande avec le tissu médiéval qui lui fait face. Il présente alors sa façade rigoureuse vers la place de Broglie, datant du XVII ${ }^{e}$ siècle, et accueillant l'hôtel de Ville et l'Opéra. Édifice important de la ville, il convient d'en comprendre les qualités architecturales car deux perspectives récentes le replacent dans 
l'actualité : le projet de candidature au Patrimoine mondial de l'UNESCO de la Neustadt et la restructuration programmée de l'édifice par le ministère de la Justice.

\section{La Justice dans la Neustadt}

3 Strasbourg n'était pas le siège d'un Parlement de province sous l'Ancien Régime et ne posséda pas de tribunal avant la Révolution française. Créé en 1790, le premier tribunal fut installé dans l'hôtel du Maréchal du Bourg, rue de la Nuée Bleue ${ }^{1}$. Les réformes judiciaires ${ }^{2}$ imposées par Napoléon aux provinces rhénanes occupées furent maintenues après leur rattachement à l'État prussien en 1815; elles marqueront profondément le système judiciaire allemand au XIX ${ }^{\mathrm{e}}$ siècle. Les lois judiciaires (Justizgesetze für das Deutsche Reich) adoptées en 1877 dans le but d'unifier les pratiques des différentes provinces rattachées au Reich, organisa quatre juridictions: Amtsgericht (tribunal d'instance), Landesgericht (tribunal de grande instance), Oberlandesgericht (cour d'appel) et Reichsgericht, la juridiction suprême de Leipzig ${ }^{3}$. Les deux premières instances siégeaient à Strasbourg mais étaient éclatées entre les trois anciens «tribunaux de paix » réunis au sein de l'Amtsgericht, et l'ancien tribunal de 1790 abritant le nouveau Landesgericht ${ }^{4}$. La volonté de les réunir au sein d'un Amts- und Landesrichtgebäude est à l'origine de l'actuel palais de justice.

Le terrain choisi au début des années 1890 se trouve en limite ouest de la Neustadt, à cheval sur l'emprise de casernes incendiées lors des bombardements de 1870 et le mur d'enceinte du XVII e siècle (entre les bastions XII et XIII) démoli après la guerre (ill. 2).

III. 2 : Plan de situation de la place impériale et de ses environs, s.d. [ca. 1880]

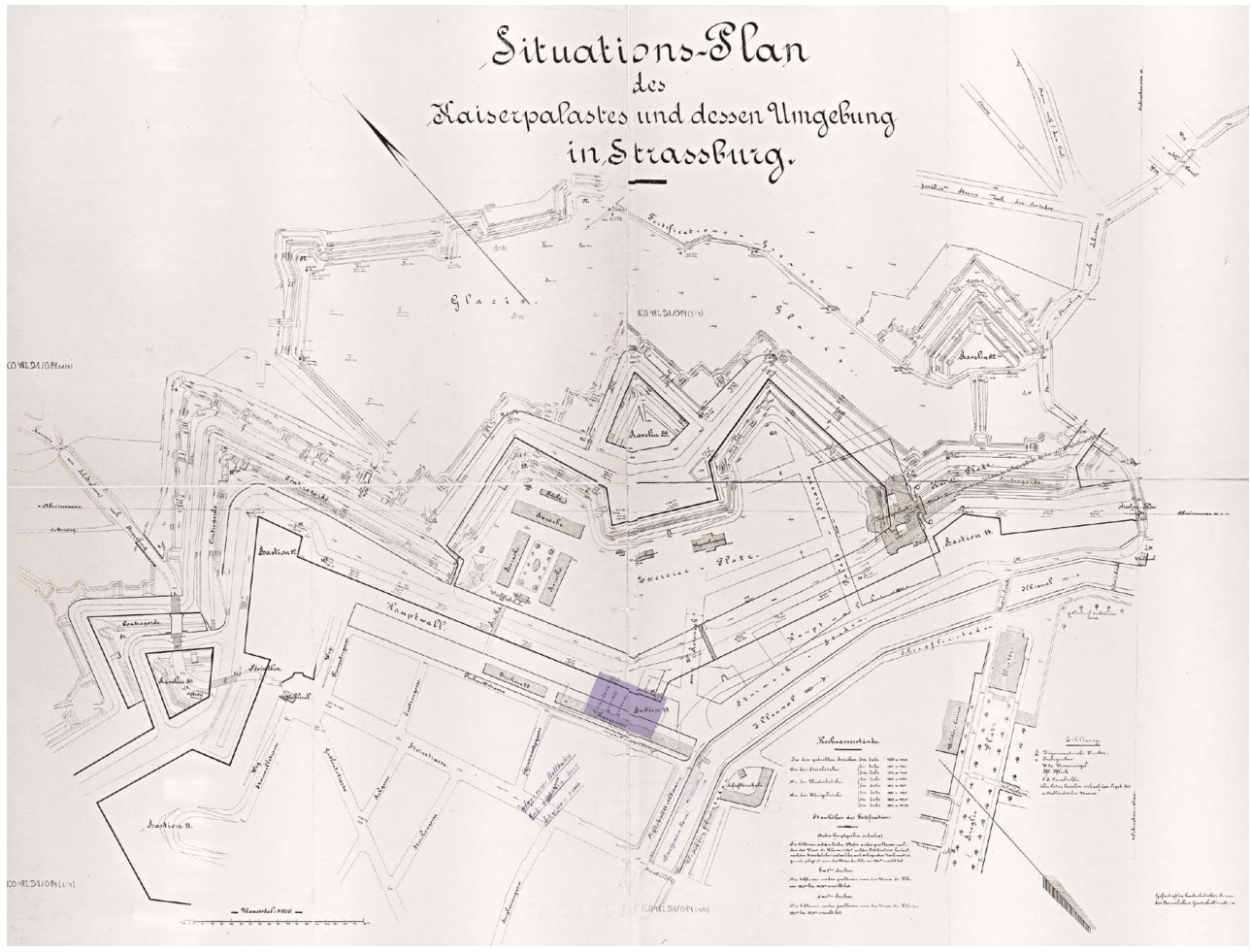

L'emprise du futur palais de justice est repérée en bleu

DRAC Alsace, Denkmalarchiv, DAR482D001-014 
Le Stadtbaumeister Johann Carl Ott ${ }^{5}$ (1846-1917) est chargé en juillet 1892 de dresser les plans du futur palais de justice, sur la base du programme ministériel définissant « l'ordonnancement des locaux, les dimensions des salles d'audience et la répartition des bureaux ${ }^{6} »$. Il conçoit également plusieurs projets de façades, de style néo-gothique et néo-renaissance inspirés de l'Hôtel du commerce (1585) de la place Gutenberg7 ${ }^{7}$. La municipalité valida les plans d'étages dressés par Ott mais ne se prononça pas sur les élévations. Un concours d'architecture pour la « conception des façades et de l'escalier "8 fut donc organisé en novembre 1893. La bibliothèque de Strasbourg conserve un jeu de plans imprimés remis selon toute vraisemblance aux candidats : les aménagements intérieurs (circulations, bureaux, salles d'audience) y sont précisément définis tandis que les parties faisant l'objet du concours (façades, vestibule et escalier d'honneur) sont laissées en blanc, la limite extérieure du projet (Grenze des Bauplatzes) étant signifiée par une ligne discontinue (ill. 3 ).

III. 3 : Palais de justice de Strasbourg, plan du rez-de-chaussée fourni lors du concours de 1893

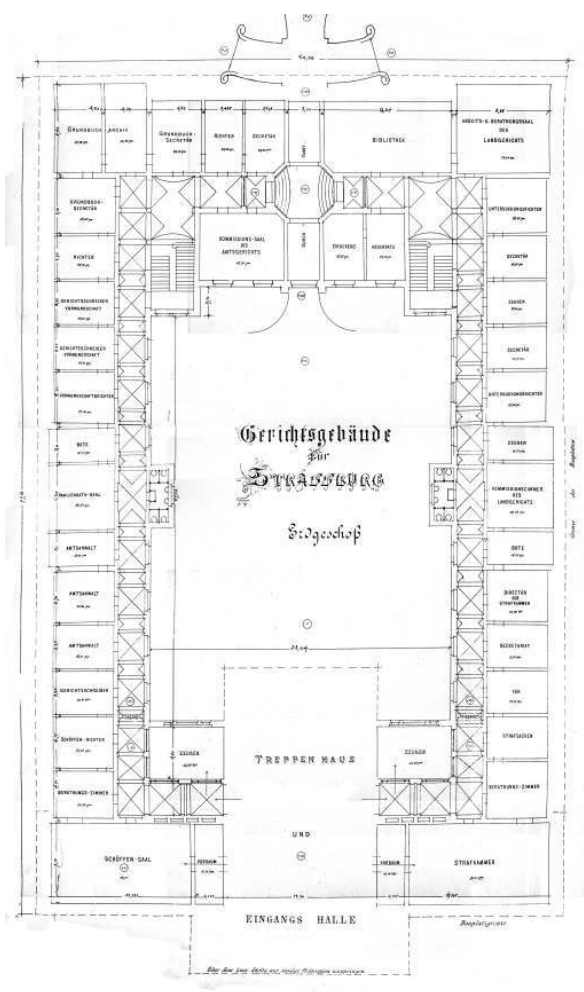

Strasbourg, BNU, M.CARTE.1.088-1

Douze projets furent reçus et le premier prix fut attribué à celui de l'architecte Skjold Neckelmann (1854-1903) dénommé Fiat justicia (ill.4). 
III. 4 : Fiat Justitia, perspective de la façade principale du projet de Skjold Neckelmann, planche de concours

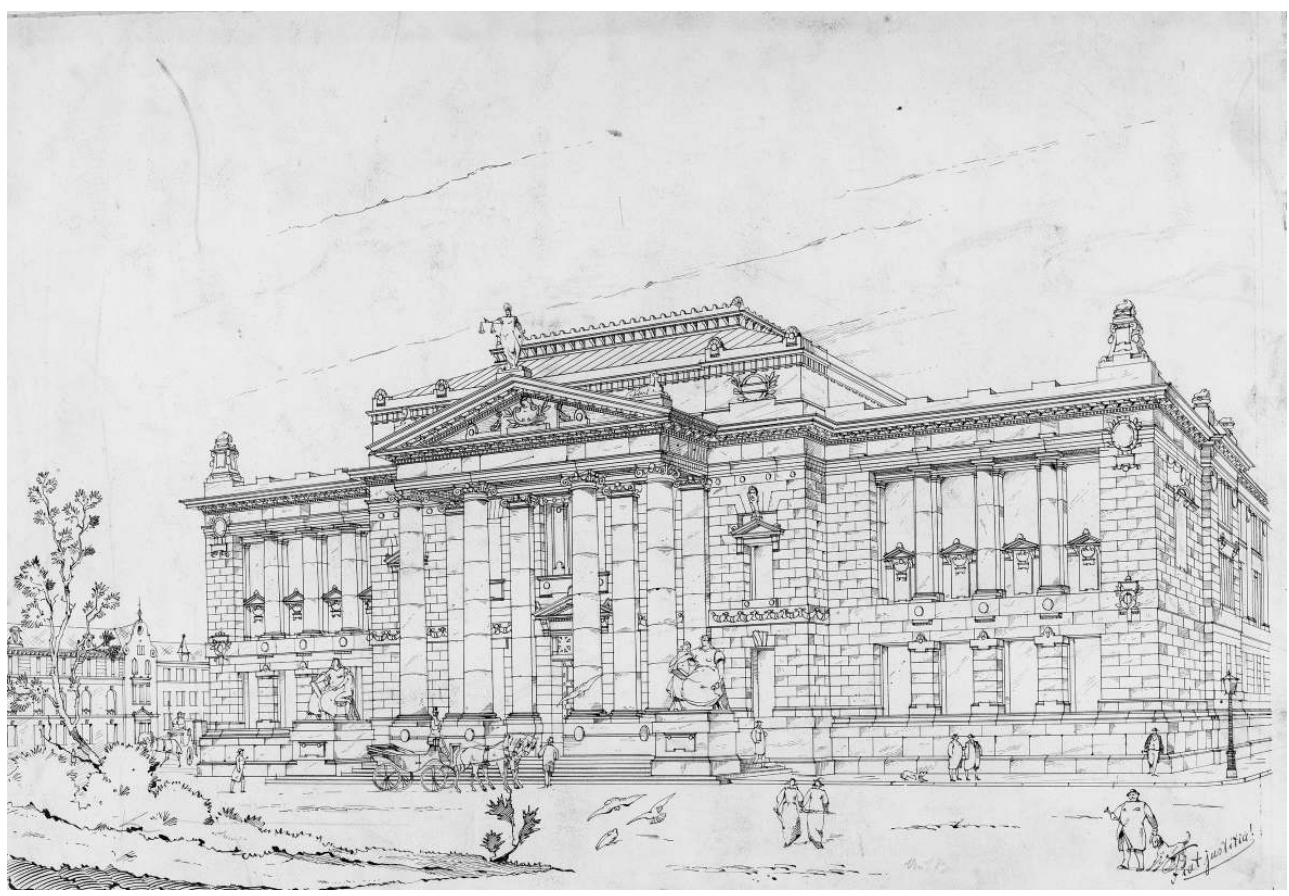

DRAC Alsace, Denkmalarchiv, DAR482B074-016

7 Implanté à Stuttgart, Neckelmann était déjà l'auteur de plusieurs édifices majeurs de la Neustadt, à commencer par la délégation de pays d'Empire (1886-1892, actuel Théâtre national) et la bibliothèque (1889-1895) sur la Kaiserplatz; en 1893 il achevait avec August Hartel l'église Saint-Pierre-le-Jeune catholique à côté du site devant le tribunal. La construction du bâtiment dura près de quatre ans: l'autorisation de construire fut accordée le 31 décembre $1894^{9}$ et le chantier ouvert en janvier $1895^{10}$; le gros œuvre était achevé à l'été 1897 et les aménagements intérieurs, un an plus tard. Le bâtiment fut inauguré le 19 septembre $1898^{11}$.

\section{Le projet de Neckelmann}

Dans le plan initial conçu par Ott, le vestibule situé à l'arrière du portique monumental d'entrée donnait directement accès aux salles d'audience du rez-de-chaussée. L'escalier ouvert sur la cour intérieure, placé dans l'axe du bâtiment, formait l'élément central de cette composition. Neckelmann, au contraire, prolonge le vestibule par une vaste salle des pas perdus (Warte Halle) dont il fait le centre symbolique et fonctionnel du projet. Le nouveau palais de justice forme un parallélépipède rectangle de 50 mètres de large et 78 mètres de long dont la façade principale se trouve sur le quai Finkmatt (ill.5). 


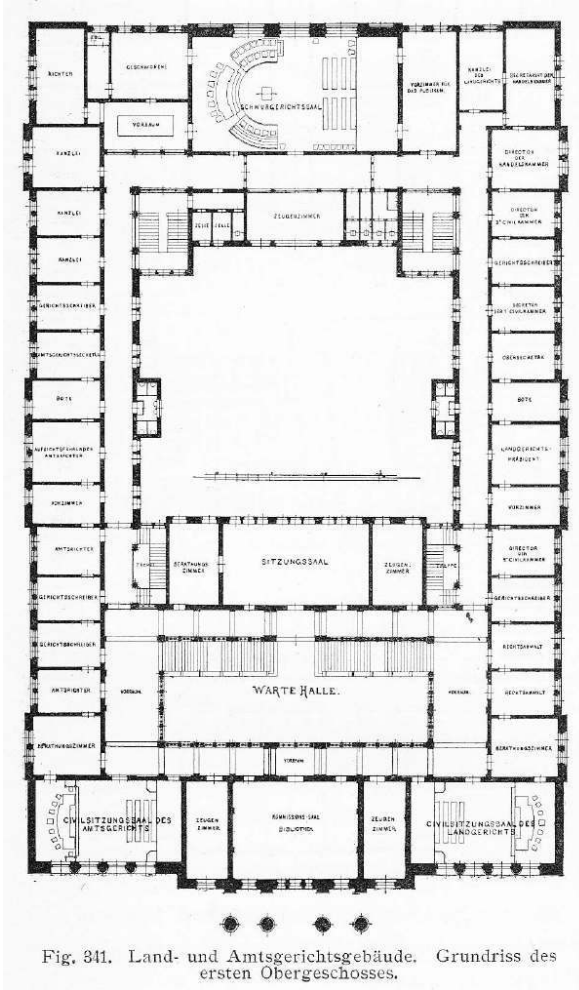

Architekten- und Ingenieur- Verein für Elsass-Lothringen, Strassburg und seine Bauten, Strasbourg, Trübner, 1894, p.432)

9 Il dispose d'une cour intérieure, accessible par l'arrière par un passage cocher. L'aile sud de l'édifice comprend un vestibule d'entrée, une salle des pas perdus et six salles d'audience (quatre seulement dans le programme initial). La salle d'assises est abritée dans l'aile nord tandis que les ailes latérales sont dédiées aux bureaux. Dès l'origine, la stricte séparation qui existe entre les espaces publics et les espaces de service facilite la gestion des flux de personnes (personnel administratif et technique, magistrats, justiciables, témoins, public) dans un lieu où elle était des plus importantes. Les deux salles d'audience ajoutées au programme ne sont peut-être pas étrangères aux modifications apportées par Neckelmann au projet de Ott : placées dans la cour au revers du hall, elles occupent l'espace initialement dévolu à l'escalier d'honneur, dès lors privé de sa position axiale et d'éclairage. La verrière zénithale, qui conduit la lumière des combles jusqu'au rez-de-chaussée, est la réponse de l'architecte à la densification du programme. Par ses dimensions (11,50 mètres de large, 28 mètres de long et 16 mètres de haut), la salle des pas perdus donne la mesure du bâtiment dont elle articule les différents niveaux (ill. 6). 


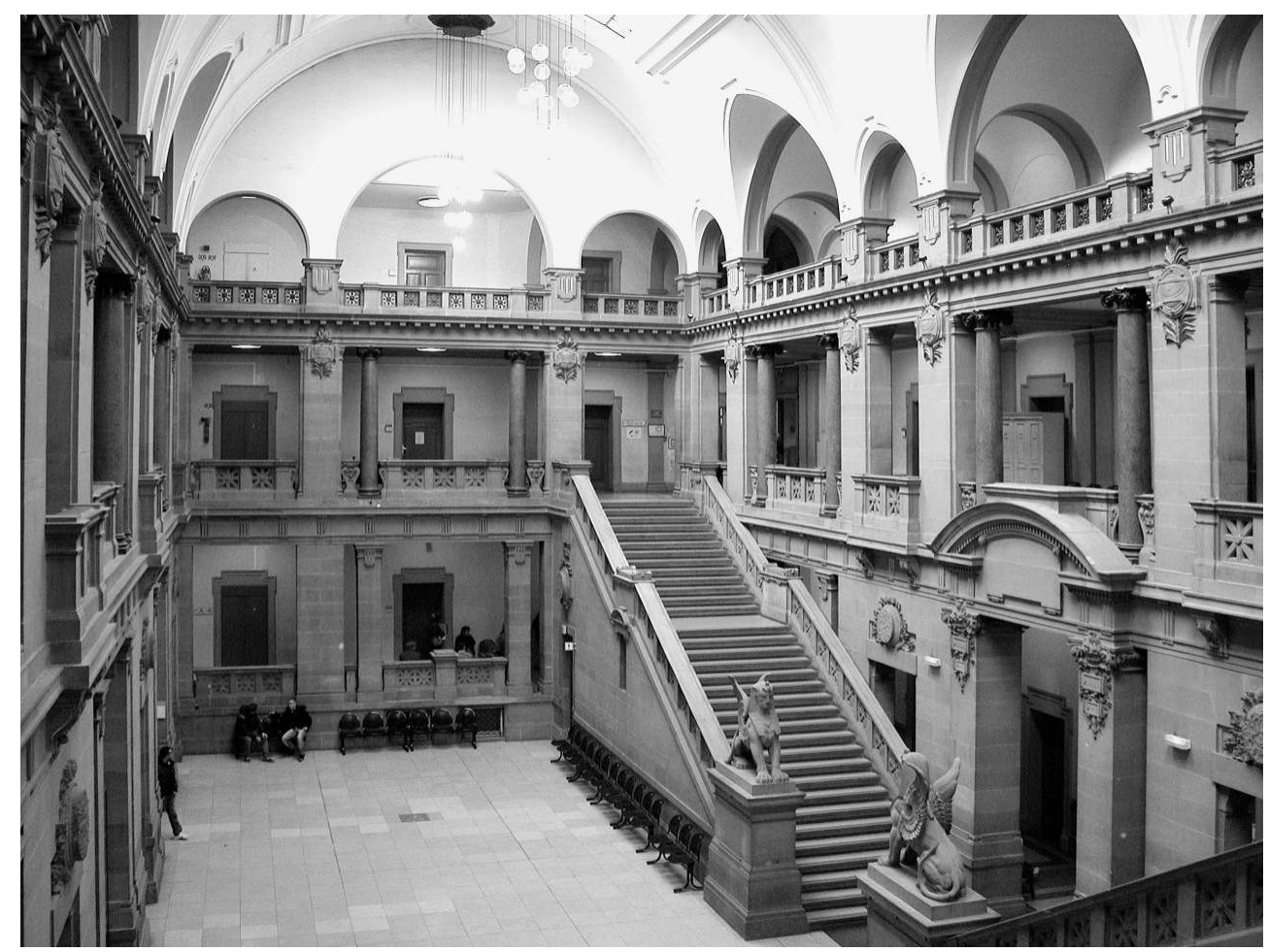

Photographie : Philippe Grandvoinnet, 2011

10 Grâce à la succession d'espaces de dimensions et de qualités diverses, Neckelmann met en scène la transition entre l'espace public urbain et l'espace de la justice: le portique à l'Antique avec ses figures allégoriques incarne la justice; resserré et, le vestibule annonce au visiteur un changement d'état ; enfin, l'espace lumineux, dilaté et structuré de la salle des Pas-Perdus achève le processus.

11 La salle d'assises, elle, située à l'arrière du bâtiment constitue la seule enclave ouverte au public dans la partie nord du bâtiment. Cette enclave permettait autrefois à cette salle de disposer de sa propre entrée (escaliers accessibles depuis le passage cocher de la façade nord) et de circulations verticales relativement indépendantes (du moins dissociables du reste de l'édifice en cas de besoins). Elle pose aujourd'hui problème dans la mesure où les règles de fonctionnement et de sécurité des palais de justice contemporains ont profondément évolué.

12 Le bâtiment dispose dès l'origine des équipements les plus perfectionnés : revêtements de sol en mosaïques colorées, granito et linoléum, réseau de chauffage central à vapeur basse pression, éclairage électrique ${ }^{12}$. Le palais de justice est à son inauguration en 1898 un équipement que l'on peut qualifier de moderne; il donnera satisfaction à l'institution qu'il abrite sans transformation notable pendant plus d'un siècle. Une surélévation partielle est toutefois réalisée à la fin des années 1970 afin d'augmenter les surfaces de plancher ${ }^{13}$. Sensible à la qualité architecturale et à la force expressive de l'ensemble, l'architecte en chef des bâtiments civils et palais nationaux en charge du projet, Roger Lamoise, limita son intervention aux ailes latérales et à la façade arrière, laissant intacte la façade principale sur le quai. 


\section{Architecture et symbole}

13 Le style officiel du Reich fondé par Bismarck emprunte ses motifs à l'architecture de la Renaissance italienne envisagée, à la suite des théories de Gottfried Semper (1803-1879), comme une «fusion organique entre le pureté des formes grecques et les techniques de construction romaine ${ }^{14} 》$. À Strasbourg, la gare (1883) et les édifices bordant la Kaiserplatz en sont les meilleurs exemples. Par ses références antiques affirmées (portique ionique grec, sphinx égyptiens, etc.), le palais de justice se démarque nettement de ce style officiel, inscrivant dans la pierre l'indépendance de l'institution judiciaire. La rigueur de sa composition fait d'ailleurs exception dans une production régionale qui échappe rarement à l'éclectisme en vigueur: Moyen Âge et Renaissance allemande pour les Amstgerichtgebäuden de Hochfelden (1895) et de Mulhouse (1902), Renaissance florentine pour le Landesgerichtgebäude de Mulhouse (1878), baroque allemand pour l' Oberlandesgerichtgebäude de Colmar (1906, actuelle cour d'appel).

Cette rigueur martiale n'est pas sans rappeler le style austère du tribunal impérial ( Reichsgerichtshaus) de Leipzig (Hoffmann et Dybwald architectes, 1884-1895), antérieur de quelques années seulement et salué en Allemagne pour la " retenue » avec laquelle fut exprimée sa monumentalité ${ }^{15}$. Il est d'ailleurs possible d'établir un parallèle entre les fonctions symboliques de ces deux projets : il s'agissait à Leipzig de sceller l'unité allemande au travers de l'institution judiciaire, à Strasbourg de réaffirmer l'ancrage de l'Alsace-Lorraine dans le Reich ${ }^{16}$. Le projet strasbourgeois puise également dans l'architecture judiciaire française de la III ${ }^{\mathrm{e}}$ République, pour laquelle le retour aux modèles grecs a une portée autant symbolique que politique. Chargé de dresser les plans du futur palais de justice, Ott visita d'ailleurs, en 1892, quelques-unes des réalisations publiées dans le Handbuch der Architektur ${ }^{17}$, en Allemagne (Francfort, Kassel, Cologne) mais aussi en France (Le Havre, Meaux). Conçu « selon l'usage antique et solennel qui veut que les palais de justice modernes se présentent à la grecque ${ }^{18}$ ", le palais de justice du Havre (1873-1876, détruit en 1944) a pu faire office de modèle, à la fois en plan, dont le schéma distributif est clair et fonctionnel, mais aussi en élévation, et jusque dans certains détails ornementaux, tels les obélisques de pierre qui flanquent le portique du tribunal de Strasbourg, utilisés au Havre comme candélabre pour l'éclairage de la façade (ill. 7). 


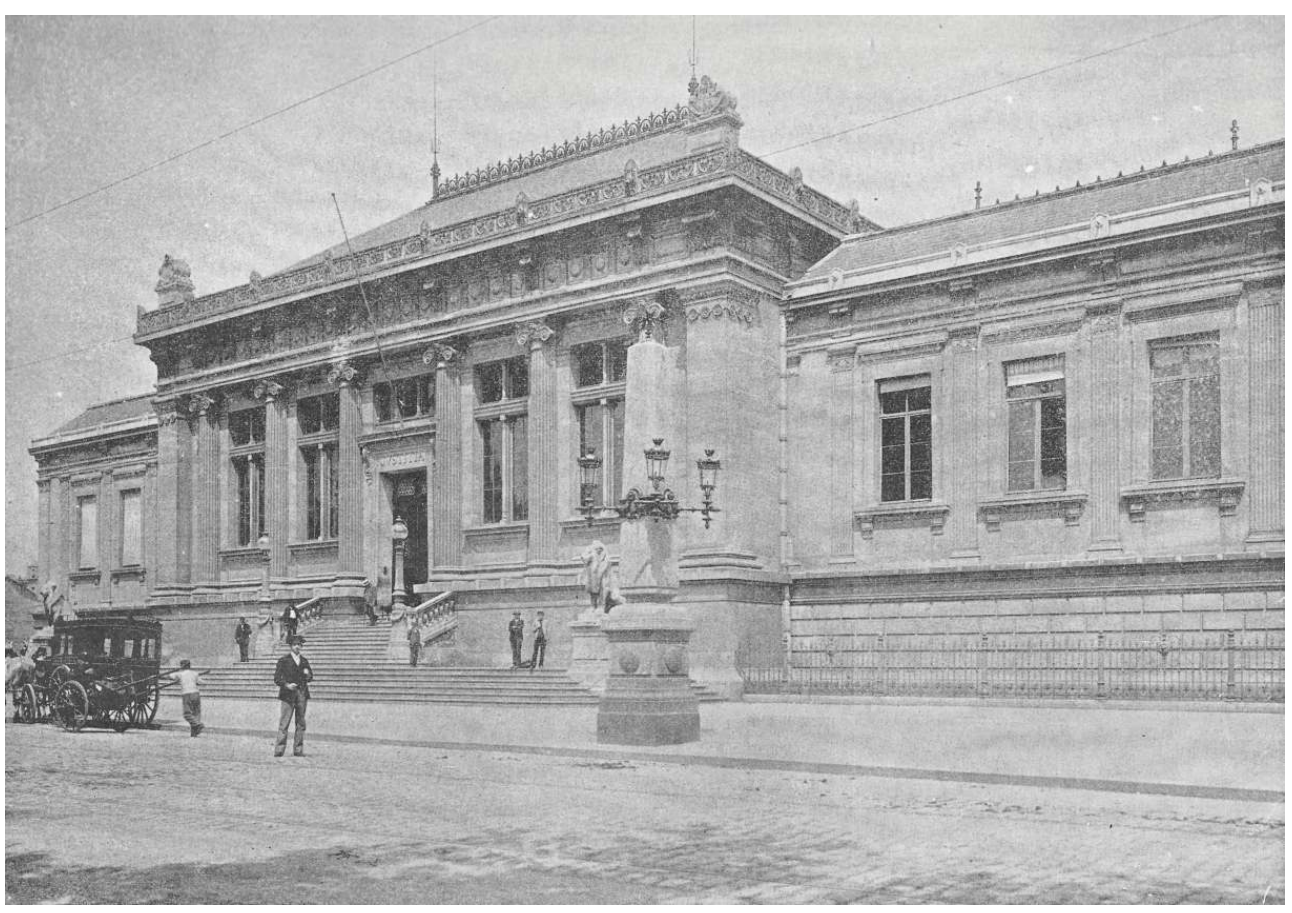

Album national : France, Algérie, Colonies. Voyage à travers la France et son empire colonial, Paris, L. Boulanger, s.d. [ca. 1900], p. 888

Dans La Justice en ses temples, Werner Szambien souligne le « caractère symbolique ${ }^{19}$ des palais de justice : ces bâtiments constituent un type architectural si bien identifié qu'il évoque à leur propos l'idée d'une "architecture parlante ${ }^{20}$ ». La distinction de deux niveaux (inférieur carcéral, supérieur judiciaire), par exemple, date du Moyen Âge et ce dispositif est rationalisé à l'époque classique : les salles d'audience occupent alors un étage noble qui repose sur un socle fermé d'où sont amenés les justiciables. À Strasbourg, l'accès des prévenus aux salles d'audience depuis le sous-sol, via la cour, s'inscrit dans cette tradition. Extérieurement, un palais de justice devait non seulement incarner l'institution judiciaire mais également donner une "idée de la justice ", d'où l'emploi d'un vocabulaire architectural chargé de sens, d'une "architecture parlante ${ }^{21}$. Ces motifs suffisent à justifier le découplage total du plan et des façades dans le processus de conception du palais de justice de Strasbourg, le premier devant optimiser le fonctionnement de l'institution judiciaire, les secondes devant exprimer les valeurs de l'institution judiciaire. Cette dissociation doit être mise en parallèle, s'agissant du monde germanique, avec les théories de l'architecte et théoricien Gottfried Semper (1803-1879) qui prônait une distinction entre la structure du bâti et son enveloppe, assimilable à un « vêtement » habillant l'édifice.

16 À Strasbourg, l'expression architecturale du palais de justice marque incontestablement la « volonté d'imposer un nouvel ordre » lié autant à la réforme portée par la loi judiciaire de 1879 qu'au nouveau statut de l'Alsace-Lorraine, devenue Reichland en 1871. Dans le Dictionnaire des Monuments historiques d'Alsace, le bâtiment est décrit comme "l'un des rares bâtiments publics strasbourgeois de cette époque totalement affranchi de la rhétorique impériale progermanique ou régionale; seules y sont admises les références 
antiques [...] réputées intemporelles et universelles, qui veulent signifier ici l'indépendance de la justice vis-à-vis de tous les pouvoirs ${ }^{22}$ ».

\section{Projet à l'œuvre}

17 Inscrit sur l'Inventaire supplémentaire en 1992 pour ses façades, la salle des Pas-Perdus et les salles d'audience et d'assises, le palais de justice de Strasbourg va connaître prochainement une restructuration lourde. Le mode de fonctionnement des palais contemporains est assez éloigné de ceux du XIX siècle. Le cahier des charges actuel recommande par exemple cinq entrées différentes pour les salles d'audience: une sécurisée pour les détenus, une pour les victimes, une pour le public, une pour le parquet et une dernière pour les magistrats du siège. Par ailleurs la justice se rend aujourd'hui dans des locaux de type bureau banalisé, pour tout ce qui concerne les affaires familiales ou le droit de l'entreprise par exemple. Pour ces raisons, le ministère de la justice a mené jusqu'à ces dernières années une politique de construction d'édifices neufs (Bordeaux, Lyon, Nantes), délaissant parfois les anciens. Les juges ont cependant fait remonter à leur ministère de tutelle le manque de solennité de ces espaces récents. À Strasbourg, face à l'impossibilité technique d'agrandir sur le site, la conservation de la fonction de justice dans les locaux actuels passe par la densification du bâtiment. Il s'agit notamment de répondre aux problématiques de flux mais aussi de trouver de la surface de bureaux rationalisés. La réflexion menée de concert par les ministères de la justice et de la culture, et à laquelle ont contribué les auteurs de l'article, a pointé notamment la problématique de la salle d'assise, qui occupe un volume important à l'écart des zones ouvertes au public. Constatant que l'objectif majeur de la sauvegarde du patrimoine est aussi de préserver les fonctions qui l'habitent, le cahier des charges qui a été établi a défini la hiérarchie des éléments pouvant faire l'objet de modifications. Si des éléments plus conventionnels ont été soulignés, comme la conservation dans son intégralité de la façade principale ou de la salle des pas perdus, d'autres ont été évoqués, comme la parfaite lisibilité fonctionnelle du plan, la qualité des espaces de distribution, l'intérêt à accorder aux éléments de décors et aux matériaux, alors que ces points étaient absents du dossier d'inscription. Le projet lauréat, de l'architecte catalan Jordi Garcés (ill.8), propose ainsi une intervention en toiture, en réinterprétant de façon contemporaine la structure pyramidale des toitures, disparue lors de la surélévation des années 1970. 


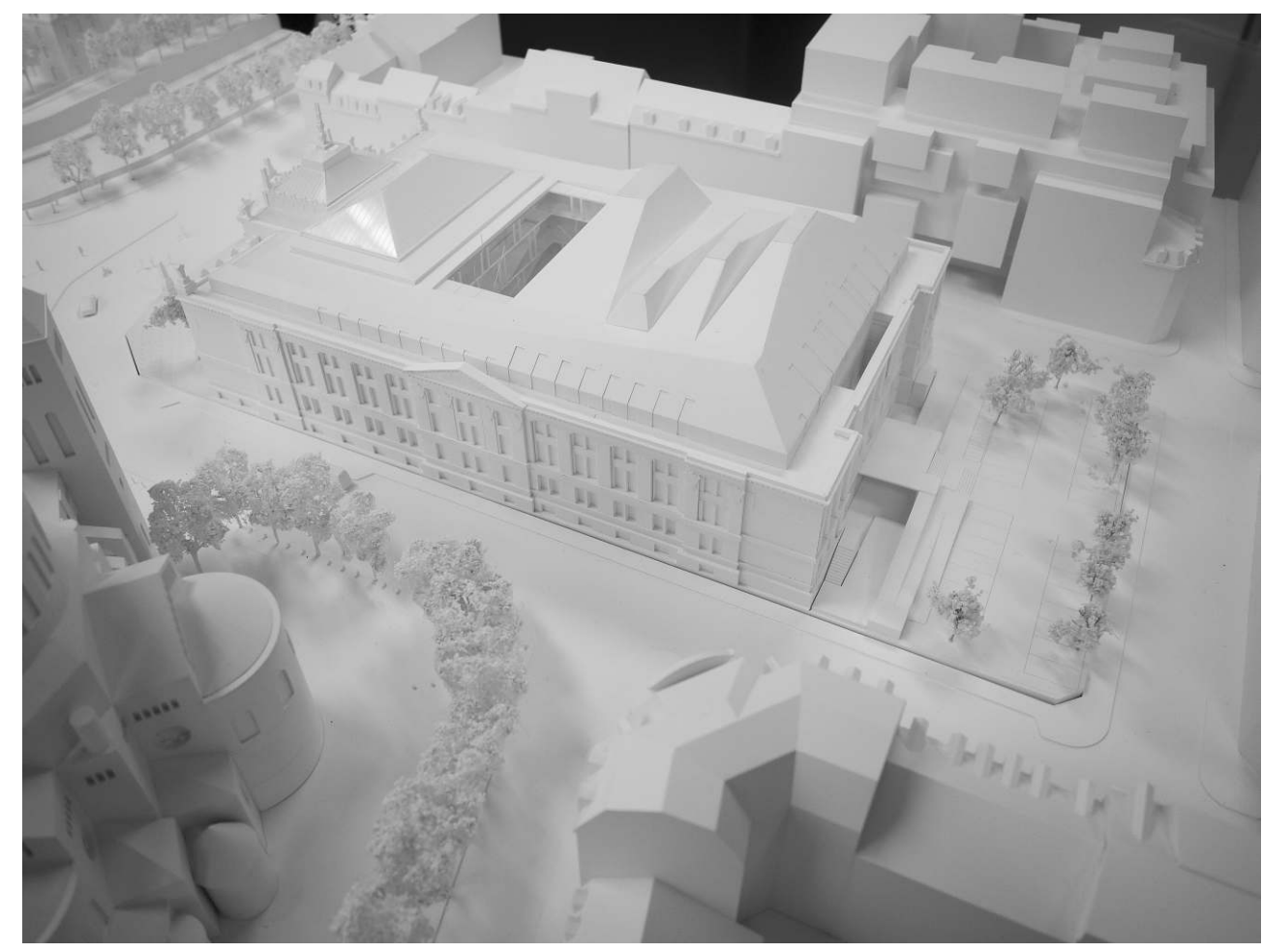

Photographie : Raphaël Labrunye, 2011

Le plan des distributions est lui aussi reconfiguré par un patio, prolongation directe de la Salle des Pas-Perdus vers les nouvelles salles d'audience. Celles-ci, incrustées au cœur du palais, dans l'ancienne cour, se situent à l'articulation des différents flux de circulation. L'aile nord et, en particulier, la salle d'assise, est profondément retouchée mais en maintenant l'intégrité des façades du palais, qui reste un édifice d'une très grande cohérence et intégrité. On pourra regretter la désaffection des anciennes salles d'audience d'origine, car les qualités de cette « architecture parlante» ne semblent pas mobilisées dans cette transformation en bureaux banalisés.

\section{NOTES}

1. Cet édifice classique construit entre 1725-1731 est déserté par son propriétaire dès le début de la Révolution. Foessel, 1974, p. 1243-1244.

2. Notamment le Code de procédure civile (1806) et le Code d'instruction criminelle (1808).

3. Klemens Klemmer, Rudolf Wassermann, Thomas Michael Wessel, Deutsche Gerichtsgebäude : von der Dorflinde über den Justizpalast zum Haus des Rechts, s.l., C.H. Beck, 1993, Munich, 184 p. p. 28.

4. Le Landesgericht sera transféré dans l'ancienne gare après la mise en service de la gare actuelle en août 1883. Une partie des locaux de la rue de la Nuée Bleue furent attribués au Conseil supérieur de l'éducation (Kaiserlicher Oberschulrath). Administration d'Alsace-Lorraine : «Vorlage 
Nr. 76, betreffend die miethweise Ueberlassung eines Theiles der Räume im hiesigen Landgerichtsgebäude, welche der Abtheilung für Justiz und Kultus des Kaiserlichen Ministeriums überlasse waren, an den Kaiserlichen Oberschulrath », 18 novembre 1887. Arch. dép. du Bas-Rhin, 84 AL 4714.

5. Johann Carl Ott (1846-1917) était architecte de la ville de Strasbourg depuis 1886, poste auquel il avait succédé à G. Conrath, auteur du plan d'extension de 1878. Wilcken, Architektur im Grenzraum, Saarbrück, Institut für Landeskunde im Saarland, 2000, p.195

6. Memorandum édité au Ministère d'Alsace-Lorraine, département de la Justice et des Cultes, 19 septembre 1898, Strasbourg, G. Fischbach, p. 3.

7. Le bâtiment abritait alors une partie de l'Amtsgericht. Wilcken, op. cit., p. 195.

8. Ibid.

9. Bürgermeisteramt der Stadt Strassburg. Baugebühren-Schein $n^{\circ} 722$, janvier 1895. Archives de la Communauté urbaine de Strasbourg, 801 W 113.

10. Les matériaux sont amenés par voie d'eau jusqu'au Stadtgraben Kanal et transportés par wagonnets sur rail jusqu'au chantier.

11. Anonyme, "Die Feier der Eröffnung des neuen Gerichtsgebäudes in Strassburg", dans Strasburger Correspondenz, 19 septembre 1898, n¹06, p. 1.

12. Les lampes à arc des salles d'audience furent toutefois rapidement remplacées des lampes à incandescence « en raison du fort bruit inévitable lors du raccordement aux lignes de courant triphasé de la centrale électrique ». Memorandum édité au ministère d'Alsace-Lorraine, département de la justice et des cultes, Strasbourg, G.Fischbach, 19 septembre 1898, p. 8.

13. Palais de Justice de Strasbourg. Aménagement des combles en bureaux. Notice de présentation et description, mai 1976, archives de la Communauté urbaine de Strasbourg. Le chantier a lieu en 1978 et 1979.

14. «Die Renaissance als eine organische Vereinigung griechischer Einzelformen mit römischer Baukunst ", dans "Der Stil in den technischen und tectonischen Künsten oder Praktische Ästhetik, 1860-1863 », cité dans Klemens Klemmer, Rudolf Wassermann, Thomas Michael Wessel, op. cit., p. 38.

15. Ibid.

16. Id, p. 62 .

17. Wilcken, op. cit., p. 195. Theodor V. Landauer, «Gerichtshäuser, Straf- und BesserungsAnstalten », dans F. Bluntschli, Gebäude für Verwaltung, Rechtspflege und Gesetzgebung ; Militärbauten , Darmstadt, A. Bergsträsser, 1887, Handbuch der Architektur ; Entwerfen, Anlage und Einrichtung der Gebäude, demi-volume ${ }^{\circ} 7$, partie 4.

18. Anonyme, Album national : France, Algérie, Colonies. Voyage à travers la France et son empire colonial , Paris, L. Boulanger, s.d. [ca. 1900], 2 vol., 1183 p., p. 888.

19. Association française pour l'histoire de la justice, La Justice en ses temples, 1992, Paris, éd. Brissaud, 323 p., p. 71.

20. W. Szambien, «Architecture parlante, architecture à caractère, architecture écrite ", dans Interférences, université de Rennes, 1988, p. 59-64.

21. Klemens Klemmer, Rudolf Wassermann, Thomas Michael Wessel, op.cit., p. 28.

22. Jean-Pierre Beck (dir.), Dictionnaire des Monuments historiques d'Alsace, Strasbourg, La Nuée bleue, 1995, 662 p., p. 515. 


\section{RÉSUMÉS}

La construction par l'administration allemande d'un nouveau palais de justice à Strasbourg (1893 - 1898) répondait à un double objectif: affirmer le statut de la ville, devenue capitale du Reichsland d'Alsace-Lorraine suite à l'annexion de 1871, et doter l'institution judiciaire d'un outil moderne du fait de la réorganisation des juridictions imposée par la loi judiciaire d'Empire de 1877 (Justizgesetze für das deutsche Reich). La légitimité de l'institution judiciaire au sein de la cité et symboliquement de la ville au sein de l'Empire - est affirmée dans le projet par l'emploi d'une architecture à l'antique, autant que par sa situation urbaine dans la Neustadt, création urbaine qui symbolise l'ambition allemande pour Strasbourg. Le palais de Justice, monument historique inscrit depuis 1992, situé dans le périmètre de la Neustadt proposé à l'inscription sur la liste du patrimoine mondial, offre ainsi une synthèse particulièrement élaborée des références allemandes et françaises en matière d'architecture judiciaire de la fin du XIX ${ }^{\mathrm{e}}$ siècle.

The construction by the German administration of a new court house in Strasbourg (1893-1898) had two goals : reinforce the town's status, capital of the Reichsland of Alsace-Lorainne since its annexation in 1871, and give the legal institution a modern tool with the reorganisation of the jurisdictions imposed by the Empire jurisdiction law of 1877 (Justizgesetze für das deutsche Reich). The legitimacy of the legal institution within the city - and symbolically of the town within the Empire - is reinforced in this project by the use of Antique style architecture, as well by its urban location in the Neustadt, urban creation which symbolises the German ambitions for Strasbourg. The court house, classified historical monument since 1992, situated in the perimetre of the Neustadt, recommended to be included on the World Heritage list, offers a particularly elaborate synthesis of references in German and French legal architecture of the end of the XIX ${ }^{\text {th }}$ century.

Der von der deutschen Verwaltung betriebene Bau eines neuen Justizpalastes in Straßburg (1893-1898) spiegelt eine doppelte Zielsetzung wider: Zum einen geht es darum, den Status der infolge der Annexion von 1871 zur Hauptstadt des „Reichslandes Elsass-Lothringen“ erklärten Stadt Straßburg zu unterstreichen, andererseits darum, den Justizapparat entsprechend der 1877 erlassenen „Justizgesetze für das Deutsche Reich“ mit angemessenen Einrichtungen zu versehen. Die Legitimität dieser Justizinstitution im Herzen der Stadt Straßburg - und darüber hinaus im neu konstituierten Deutschen Reich - wird ebenso durch den Einsatz antiker Motive unterstrichen wie auch durch die Einbettung des Baus in die Straßburger „Neustadt", die als städtebauliche Neuschöpfung die urbanistischen Pläne der deutschen Verwaltung widerspiegelt. Der seit 1992 als Denkmal verzeichnete Justizpalast, der sich innerhalb der derzeit als Erweiterung des Weltkulturerbes vorgeschlagenen Straßburger „Neustadt“ befindet, verkörpert eine besonders ausgefeilte Synthese französischer und deutscher Gerichtsarchitektur des ausgehenden 19. Jahrhunderts. 


\section{AUTEURS}

\section{PHILIPPE GRANDVOINNET}

Philippe GRANDVoINNET est architecte et docteur en architecture, chercheur associé à l'École nationale supérieure d'architecture de Versailles et à l'Université de Strasbourg (laboratoire Arts, civilisation et histoire de l'Europe). Formé à la sauvegarde du patrimoine bâti moderne et contemporain auprès du Pr. Bruno Reichlin à l'Institut d'architecture de l'Université de Genève, il est l'auteur de nombreuses publications sur l'architecture et le patrimoine du XX $\mathrm{XX}^{\mathrm{e}}$ siècle. Architecte et urbaniste de l'État au ministère de la culture et de la communication depuis 2012, il occupe un poste d'architecte des bâtiments de France, chef du service territorial de l'architecture et du patrimoine des Hautes-Alpes, à Gap. Adresse électronique : philippe.grandvoinnet (at) culture.gouv.fr.

\section{RAPHAËL LABRUNYE}

Raphaël LABRUNYE est architecte diplômé par le gouvernement et docteur en histoire. Il mène une activité d'études patrimoniales et de maîtrise d'œuvre au sein de son agence Architecture \& Patrimoine. Maître assistant associé à l'École nationale supérieure d'architecture de Normandie, il poursuit son travail de recherche au sein de l'équipe ATE (Architecture Territoire Environnement) autour des questions posées par l'intervention sur les ensembles de logements de l'après-guerre (recherche pour le ministère de la culture et de la communication, avec Benoît Carrié). Adresse électronique : r.labrunye (at) gmail.com 